\title{
Prognostic value of preoperative absolute lymphocyte count in recurrent hepatocellular carcinoma following thermal ablation: a retrospective analysis
}

\author{
This article was published in the following Dove Press journal: \\ OncoTargets and Therapy \\ 3 October 2014 \\ Number of times this article has been viewed
}

Xin Li

Zhiyu Han

Zhigang Cheng

jie Yu

Xiaoling Yu

Ping Liang

Department of Interventional Ultrasound, Chinese PLA General Hospital, Beijing, People's Republic of China
Correspondence: Ping Liang

Department of Interventional Ultrasound, Chinese PLA General Hospital, 28 Fuxing Road, Beijing 100853, People's Republic of China

Tel +86 I0 66939530

Fax +86 106816 I218

Email liangping30I@hotmail.com
Purpose: To investigate the prognostic value of preoperative absolute lymphocyte count (ALC) in recurrent hepatocellular carcinoma (RHCC) following thermal ablation.

Materials and methods: We retrospectively analyzed the relationship between preoperative ALC and the clinicopathologic factors and long-term prognosis in 423 RHCC patients who underwent curative thermal ablation. Correlation analysis, receiver operating characteristic (ROC) calculation, Kaplan-Meier curves, and multivariate regression were used for statistical analysis.

Results: The median time to recurrence was 12 months for RHCC patients after thermal ablation. On multivariate Cox regression analysis, preoperative ALC was an independent risk factor for cancer recurrence, along with tumor differentiation and $\alpha$-fetoprotein level. ALC $\geq 1.64 \times 10^{9} / \mathrm{L}$ defined by ROC calculation was associated with prolonged survival (area under the curve 0.741 , $P<0.001)$. Patients with ALC $\geq 1.64 \times 10^{9} / \mathrm{L}$ showed a mean survival of 20.2 months versus 11.6 months for patients with ALC $<1.64 \times 10^{9} / \mathrm{L}(P<0.001)$. Patients were stratified into high and low groups according to ALC status. After excluding the basic parameters between groups, the 1- and 3-year recurrence rates in the high group were $20.9 \%$ and $29.5 \%$, respectively, which were significantly lower than those of the low group ( $58.4 \%$ and $71.9 \%$, respectively; $P<0.001$ ). The recurrence-free survival rates in the two groups analyzed by Kaplan-Meier curves were significantly different $(P<0.001)$.

Conclusion: Preoperative ALC is a powerful prognostic factor for RHCC recurrence after thermal ablation, which suggests that maintaining a high ALC in RHCC patients might improve cancer outcomes.

Keywords: absolute lymphocyte count, recurrent hepatocellular carcinoma, thermal ablation, recurrence

\section{Introduction}

Hepatocellular carcinoma ( $\mathrm{HCC}$ ) is one of the most aggressive and prevalent malignant diseases worldwide, and is characterized by a dismal prognosis. ${ }^{1} \mathrm{HCC}$ most commonly arises in patients with chronic liver disease, which induces chronic inflammation and impaired immunity. ${ }^{2,3}$ The high recurrence and metastasis rate after radical treatment are the main hurdles for improving HCC prognosis. ${ }^{4,5}$ For recurrent HCC (RHCC) patients, immune function and general health is impaired, and thus surgical resection may not be the first treatment choice. Instead, thermal ablation, a less invasive option that provides excellent clinical results, should be considered..$^{6,7}$ 
Absolute lymphocyte count (ALC) is considered a surrogate marker for the level of immunosuppression and nutritional status of patients, and it is a prognostic factor for survival and recurrence in several cancers, including HCC with hepatitis. ${ }^{8,9}$ Therefore, despite being a relatively crude measure, ALC serves as a useful barometer of immune function and general health in humans. ${ }^{10}$ Cancer patients also frequently show decreased ALC at diagnosis. In 1970, Riesco reported that ALC was positively associated with the "curability" of a variety of cancers. ${ }^{11}$ Similar associations between ALC and survival have been reported for a wide variety of epithelial, connective tissue, and lymphoid cancers, ${ }^{12}$ including HCC after liver transplantation. ${ }^{9}$ The liver itself is an immunological organ, and the immune system strongly influences outcomes in patients with $\mathrm{HCC} .{ }^{13} \mathrm{ALC}$ as a prognostic factor for primary $\mathrm{HCC}$ after liver transplantation or resection has been reported, but it has not been extensively evaluated in patients with RHCC treated by thermal ablation.

The aim of this retrospective study was to determine the best cutoff value for preoperative ALC in RHCC patients after thermal ablation and to evaluate whether the new ALC cutoff point correlates with tumor recurrence. Furthermore, we established a simple preoperative prognostic score model suggesting that maintaining ALC in RHCC patients might improve cancer outcomes.

\section{Materials and methods Patient population}

From May 2006 to May 2014, 423 RHCC patients (351 males and 72 females, mean age $59.4 \pm 11.0$ years, range $28-82$ years) with 989 HCC nodules detected on contrast-enhanced ultrasound/computed tomography/magnetic resonance imaging (CEUS/CT/MRI) were enrolled in this study. All patients underwent thermal ablation with curative intention at our institution, and the protocol was designed in accordance with the patient's medical history, CT/MRI, and laboratory examination results. The maximum diameter of the lesions ranged from 1.0 to $6.0 \mathrm{~cm}$ (mean \pm standard deviation [SD], $2.6 \pm 1.3 \mathrm{~cm}$ ). Curative pretreatment included liver resection $(\mathrm{n}=75)$, transarterial chemoembolization (TACE; $n=150$ ), and thermal ablation ( $\mathrm{n}=198$ ). Infection-virus types were as follows: hepatitis B virus (HBV)-infected, 328; HCV-infected; 41, HBV + HCVinfected, 9; and no infection, 45. The Child-Pugh score of the RHCC patients was evaluated as A level $(n=403)$ and B level $(n=20)$. ALC was defined as the absolute neutrophil count multiplied by the percentage of lymphocytes. The final HCC diagnosis was verified by pathological examination. This study was approved by the Medical Ethics Committee of Chinese
PLA General Hospital. Written informed consent was obtained from every patient.

\section{Preablation examination}

The inclusion criteria for our study were as follows: 1) nonresectable tumors or patient refusal to undergo surgery, 2) single HCC lesion $\leq 6 \mathrm{~cm}, 3$ ) more than two HCC lesions with a maximum diameter $\leq 4 \mathrm{~cm}, 4$ ) the absence of portal vein thrombosis or extrahepatic metastases, 5) prothrombin time (PT) $<25$ seconds, 6) prothrombin activity $>40 \%$, and 7) platelet count $>40$ cells $\times 10^{9} / \mathrm{L}$. Exclusion criteria were: 1) severe cardiopulmonary disease, 2) serious renal function failure, 3), severe liver-function failure, such as large-volume ascites or hepatic encephalopathy, and 4) active severe infection. All patients had undergone conventional ultrasound, CEUS, CT (computer tomography) and/or MRI (magnetic resonance imaging) to delineate the target tumor before ablation.

\section{Microwave-ablation protocol}

All treatments were performed at our institution. Before ablation, US/CEUS-guided biopsy was performed first using an automatic biopsy gun with an $18 \mathrm{G}$ cutting needle under local anesthesia with $1 \%$ lidocaine; two or three punctures were performed. Subsequently, the antennas were percutaneously inserted into the tumor and placed in the desired location by US or CEUS guidance. For lesions $<1.7 \mathrm{~cm}$ in diameter, a single antenna was used; for those $\geq 1.7 \mathrm{~cm}$ in diameter, we used two or more antennas. General anesthesia (propofol, $6-12 \mathrm{mg} / \mathrm{kg}$ per hour; ketamine, $1-2 \mathrm{mg} / \mathrm{kg}$ ) was employed after correctly placing the antennas, and ablation was implemented. If the lesion was adjacent to the bile duct, gallbladder, or bowel $(\leq 5 \mathrm{~mm}$ ), a $21 \mathrm{G}$ thermocouple was placed percutaneously at a designated location to monitor temperature in real time. ${ }^{14}$ The temperature was kept at $50^{\circ} \mathrm{C}-54^{\circ} \mathrm{C}$ for no longer than 3 minutes, with intermittent emission of microwaves.$^{15}$ If the lesion was near the diaphragmatic dome, artificial ascites were used. In order to perform complete ablation of lesions larger than $5 \mathrm{~cm}$ or very close to the bile duct, gallbladder, or bowel, ethanol was injected into the marginal tissue of the tumor through a $21 \mathrm{G}$ percutaneous transhepatic cholangiography needle during ablation. When the hyperecho overlapped the whole lesion, the antennas were withdrawn. During the process, the needle tracks were routinely cauterized to avoid bleeding and tumor seeding.

\section{Follow-up}

Complete ablation was defined as the absence of enhancement in any areas of the lesion on enhanced images obtained at 
1 month after thermal ablation. The follow-up period started and continued until May 2014, ranging from 12 to 96 months. During the follow-up period, routine CEUS and CT and/or MRI were repeated to monitor for recurrence or metastasis at 3 months after ablation, and then at 6-month intervals. Once both $\mathrm{CE}$ images were positive or US-guided core needle biopsy was positive, the follow-up was terminated.

\section{Statistical analysis}

Data were analyzed using SPSS for Windows (version 17.0; SPSS Inc., Chicago, IL, USA). All data are expressed as mean \pm SD. Preoperative clinical parameters that impacted recurrence-free survival (RFS) were entered into the multivariate Cox proportional-hazard model to determine their independent effects. A best cutoff value of preoperative ALC was determined by time-dependent receiver-operating characteristic (ROC) curve analysis. ${ }^{16}$ Independent $\chi^{2}$ tests were used to compare categorical variables. Continuous variables were compared using unpaired $t$-tests. RFS curves were analyzed using the Kaplan-Meier method and compared using the logrank test. $P<0.05$ was considered statistically significant.

\section{Results}

\section{Correlation between ALC and RHCC recurrence following thermal ablation}

To determine whether ALC was correlated with cancer recurrence after thermal ablation in RHCC patients, we performed multivariate Cox proportional-hazard model analysis. The statistically significant predicative factors for recurrence identified are shown in Table 1. Among the 12 clinical parameters (pretreatment, age, sex, tumor number, size of tumor, type of hepatitis, cirrhosis, tumor differentiation, PT, ALC, and Child-Pugh score), low tumor differentiation, high $\alpha$-fetoprotein, low PT, and low ALC were all independent predicative factors for cancer recurrence in RHCC patients.

\section{Selection of the best cutoff value for ALC in RHCC patients}

To analyze the predicted value of ALC for cancer recurrence in RHCC patients following thermal ablation, a time-dependent ROC curve was generated. An ALC of $1.64 \times 10^{9} / \mathrm{L}$ was the best cutoff point for predicting recurrence after thermal ablation in RHCC patients (area under the ROC was 0.741 , $P<0.001$; Figure 1). We therefore utilized an ALC cutoff of $1.64 \times 10^{9} / \mathrm{L}$ as a risk factor for RHCC recurrence. All patients were divided into one of two groups: a low $\left(<1.64 \times 10^{9} / \mathrm{L}\right)-$ ALC group $(n=293,69.3 \%)$ and a high $\left(\geq 1.64 \times 10^{9} / \mathrm{L}\right)-\mathrm{ALC}$ group ( $n=130,30.7 \%)$. This result further indicates that the majority of RHCC patients had impaired immunity status, which may reduce the antitumor capabilities of RHCC patients and may be the basis of their greater inclination for recurrence.

\section{Comparisons of recurrence rates in low- and high-ALC groups}

During the follow-up period, the 1- and 3-year recurrence rates were $58.4 \%$ and $71.9 \%$ in the low-ALC group, respectively, which were significantly higher than those in the high-ALC group (20.8\% and $29.5 \%$, respectively; $P<0.001)$. Therefore, ALC is a good predictor of the risk of cancer recurrence in RHCC patients following thermal ablation.

Table I Multivariate Cox regression analysis of clinical factors in relation to recurrence-free survival of recurrent hepatocellular carcinoma patients

\begin{tabular}{|c|c|c|c|c|c|c|c|c|}
\hline \multirow[t]{2}{*}{ Clinical parameters } & \multirow[t]{2}{*}{ B } & \multirow[t]{2}{*}{ SE } & \multirow[t]{2}{*}{ Wald } & \multirow[t]{2}{*}{$d f$} & \multirow{2}{*}{$\begin{array}{l}\text { Significance } \\
\text { (P-value) }\end{array}$} & \multirow[t]{2}{*}{$\operatorname{Exp}(B)$} & \multicolumn{2}{|c|}{$95 \% \mathrm{Cl}$ for $\exp (\mathrm{B})$} \\
\hline & & & & & & & Lower & Upper \\
\hline Pretreatment & 0.118 & 0.091 & 1.679 & 1 & 0.195 & 1.125 & 0.942 & $\mathrm{I} .344$ \\
\hline Sex & 0.294 & 0.186 & 2.504 & 1 & 0.114 & 0.745 & 0.518 & 1.073 \\
\hline Age & -0.004 & 0.006 & 0.381 & 1 & 0.537 & 0.996 & 0.985 & 1.008 \\
\hline Tumor numbers & 0.020 & 0.061 & 0.110 & 1 & 0.740 & 10.020 & 0.905 & 1.150 \\
\hline Size of tumors & 0.033 & 0.048 & 0.474 & 1 & 0.491 & 0.967 & 0.880 & 1.063 \\
\hline Differentiation & 0.434 & 0.099 & 19.064 & 1 & $0.000^{*}$ & 1.543 & 1.270 & 1.875 \\
\hline Cirrhosis & 0.331 & 0.019 & 3.026 & 1 & 0.082 & 0.718 & 0.494 & 1.043 \\
\hline Type of hepatitis & 0.023 & 0.079 & 0.081 & I & 0.775 & 0.978 & 0.837 & 1.142 \\
\hline $\operatorname{AFP}(\mu g / L)$ & 0.000 & 0.000 & 5.486 & 1 & $0.019 *$ & 1.000 & 1.000 & 1.000 \\
\hline ALC & -1.064 & 0.126 & 71.200 & 1 & $0.000 *$ & 0.345 & 0.270 & 0.442 \\
\hline PT & 0.045 & 0.024 & 3.644 & I & 0.056 & 1.046 & 0.999 & 1.095 \\
\hline Child-Pugh & -0.570 & 0.311 & 3.356 & I & 0.067 & 0.566 & 0.307 & $1.04 \mid$ \\
\hline
\end{tabular}

Note: $* p<0.05$.

Abbreviations: SE, standard error; Cl, confidence interval; AFP, $\alpha$-fetoprotein; ALC, absolute lymphocyte count; PT, prothrombin time; df, degrees of freedom. 


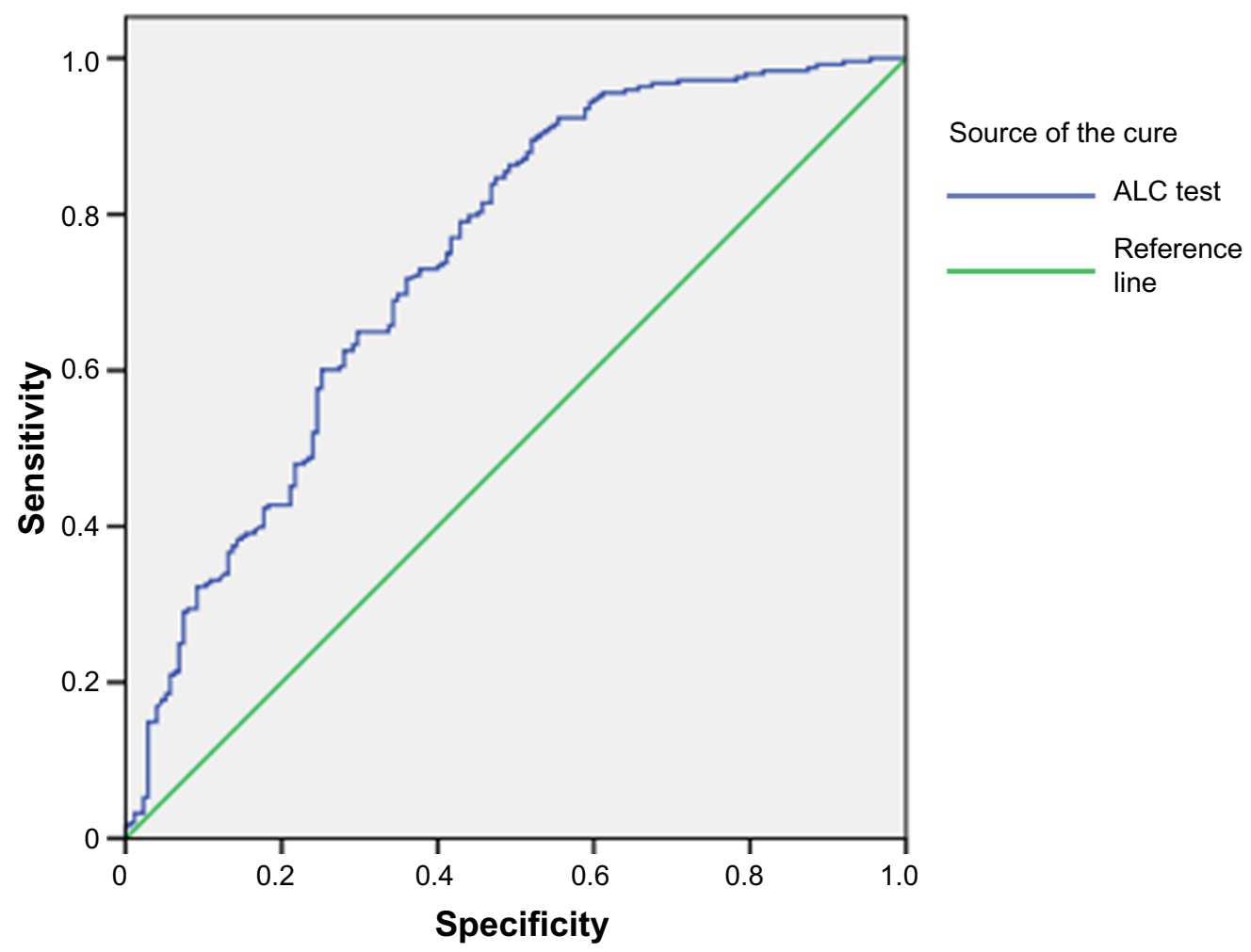

Figure I Receive-operating characteristic curve (ROC) analysis for ALC predicts recurrence in RHCC patients following thermal ablation. Notes: $A L C \geq 1.64 \times 10^{9} / \mathrm{L}$ was determined to be the cutoff value (AUROC $=0.74 \mathrm{I}, P<0.00 \mathrm{I}$ ). Diagonal segments are produced by ties. Abbreviations: ALC, absolute lymphocyte count; RHCC, recurrent hepatocellular carcinoma; AUROC, area under the ROC.

\section{Comparisons of RFS in low- and high-ALC groups}

To compare the differences in RFS rates between the lowALC and high-ALC groups, we first evaluated the basic clinical parameters between the two groups; of the eleven clinical parameters (Tables 2 and 3), none differed significantly between the low-ALC and high-ALC group. The RFS rate after thermal ablation was $28.3 \%$ in the low-ALC group and $70.4 \%$ in the high-ALC group $(P<0.001)$. The RFS rates of patients in the low- and high-ALC groups are shown in Figure 2. The RFS rate was significantly higher in the low-ALC group than in the high-ALC group $(P<0.001)$.

\section{Discussion}

In this retrospective study, preoperative ALC, tumor differentiation, and high $\alpha$-fetoprotein levels were associated with cancer recurrence in RHCC patients after thermal ablation. These results suggest that preoperative ALC is an independent, powerful, and predictive factor for cancer recurrence of RHCC following thermal ablation. As reported in several studies, ALCs have been associated with favorable prognosis in many cancers and metastases. ${ }^{10,12,17}$
Therefore, it is reasonable to state that ALC may be a simple and effective marker for determining the risk for recurrence in RHCC patients.

However, it has been reported that the ALC cutoff values for predicting prognosis and recurrence vary in different cancers. ${ }^{10,12,18}$ To our knowledge, this is the first study analyzing the best ALC cutoff value for predicting cancer recurrence in RHCC patients. On analyzing the ROC curve analysis, the best cutoff value $\left(1.64 \times 10^{9} / \mathrm{L}\right)$ was found to be discriminating for predicting cancer recurrence in RHCC patients following thermal ablation. The first interesting observation is that the incidence of ALC $<1.64 \times 10^{9} / \mathrm{L}$ in RHCC patients was very high $(69.3 \%)$. This phenomenon further indicates that the immunity status of RHCC patients has been impaired. These observations strongly suggest that low ALC is related to cancer recurrence and prognosis.

In this study, we compared the 1- and 3-year recurrence rates of the high- and low-ALC groups, and observed a marked and significant difference between the two groups. Furthermore, we compared the RFS of these two groups, and we also found a marked and significant difference. ALC is considered a surrogate marker for the level of 
Table 2 Comparison of clinical parameters of recurrent hepatocellular carcinoma patients between the low- and high-ALC groups

\begin{tabular}{|c|c|c|c|c|}
\hline Clinical parameters & $\mathbf{n}$ & $\begin{array}{l}\text { ALC } \geq 1.64 \times 10^{9} / L \\
(n=130)\end{array}$ & $\begin{array}{l}\text { ALC }<1.64 \times 10^{9} / L \\
(n=293)\end{array}$ & $P$-value \\
\hline Pretreatment & & & & 0.815 \\
\hline Surgery & 75 & 24 & 51 & \\
\hline TACE & 150 & 43 & 107 & \\
\hline Ablation & 198 & 63 & 137 & \\
\hline Sex & & & & 0.328 \\
\hline Male & 351 & 110 & $24 I$ & \\
\hline Female & 72 & 20 & 52 & \\
\hline Type of hepatitis & & & & 0.703 \\
\hline HBV & 328 & 105 & 223 & \\
\hline $\mathrm{HCV}$ & 41 & 11 & 30 & \\
\hline $\mathrm{HBV}+\mathrm{HCV}$ & 9 & 3 & 6 & \\
\hline No & 45 & 11 & 34 & \\
\hline Differentiation & & & & 0.116 \\
\hline High & 235 & 82 & 153 & \\
\hline Middle & 153 & 39 & 114 & \\
\hline Low & 35 & 9 & 26 & \\
\hline Child-Pugh & & & & 0.09 \\
\hline A & 403 & 127 & 276 & \\
\hline B & 20 & 3 & 17 & \\
\hline Cirrhosis & & & & 0.067 \\
\hline Yes & 357 & 104 & 253 & \\
\hline No & 66 & 26 & 40 & \\
\hline
\end{tabular}

Abbreviations: ALC, absolute lymphocyte count; TACE, transcatheter arterial chemoembolization; HBV, hepatitis B virus; HCV, hepatitis C virus.

immunosuppression and nutritional status of a patient, and it is also a prognostic factor for survival and recurrence in several cancers. This result further verifies that immunosuppression is common in RHCC patients, which is similar to other cancers. ${ }^{19-21}$

An ALC reduction in patients may not only be a parameter correlated with survival but also a biological mechanism stimulating tumor progression. Immunity in the human body is a balance, and once this balance is disrupted, a tumor can develop and progress. ${ }^{22,23}$ Therefore, it is very important to explore the mechanisms underlying the association between low lymphocyte count and poor prognosis. It has been reported that the possible mechanism may be as follows: 1) the low lymphocyte count may be associated with a preexisting immunosuppressed condition, suggesting that the host tends to have an inadequate immunological response; 2) the low lymphocyte count may be a consequence of lympholytic cytokines produced by lymphoma cells, and this lymphoma may itself be resistant; 3 ) a combination of both or other factors; ${ }^{12}$ or 4 ) the composition and proportion of lymphocytes may have changed. Effectively, the mechanism of low ALC in cancer patients remains unclear, but it is probably multifactorial.

There are several limitations to this study. For instance, this was a retrospective study with only a small number of patients enrolled. Moreover, the mechanism underlying the relationship between ALC and cancer recurrence in RHCC patients was not elucidated. Thirdly, the composition and proportion of lymphocytes should be further analyzed. Finally, multicenter prospective studies are needed to confirm and update the findings demonstrated in this study.

In conclusion, our results suggest that RHCC patients with low ALC have poor RFS following thermal ablation. The best ALC cutoff value was $1.64 \times 10^{9} / \mathrm{L}$ for predicting cancer recurrence, which may improve the clinical management of RHCC patients after thermal ablation. Preoperative ALC is a simple and powerful prognostic factor for the recurrence of RHCC after thermal ablation, which suggests

Table 3 Comparison of clinical parameters of recurrent hepatocellular carcinoma patients between the low- and high-ALC groups

\begin{tabular}{llllll}
\hline Group & Age (years) & Number of tumors & Size of tumors $(\mathbf{c m})$ & AFP $(\mu \mathrm{g} / \mathrm{L})$ & PT $(\mathrm{seconds})$ \\
\hline $\mathrm{ALC} \geq 1.64 \times 10^{9} / \mathrm{L}$ & $57.5 \pm 11.6$ & $2.3 \pm 1.1$ & $2.6 \pm 1.3$ & $559.4 \pm 2,816.9$ & $14.1 \pm 2.9$ \\
$\mathrm{ALC}<1.64 \times 10^{9} / \mathrm{L}$ & $60.1 \pm 10.5$ & $2.0 \pm 1.0$ & $2.5 \pm 1.2$ & $479.8 \pm 2,292.1$ & $14.6 \pm 2.6$ \\
P-value & 0.058 & 0.916 & 0.471 & 0.897 & 0.078 \\
\hline
\end{tabular}

Abbreviations: ALC, absolute lymphocyte count; AFP, $\alpha$-fetoprotein; PT, prothrombin time. 


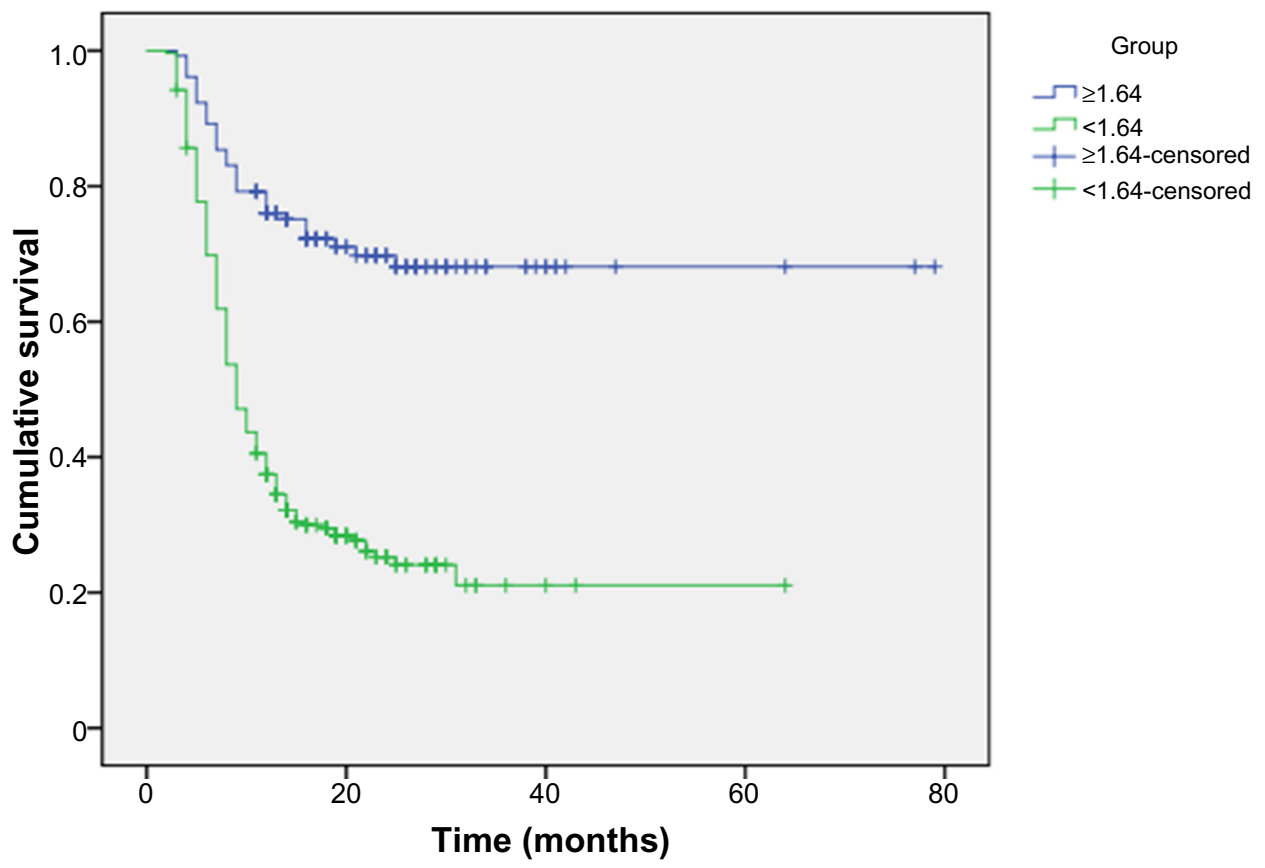

Figure 2 Comparison of RFS rates in the low $\left(<1.64 \times 10^{9} / \mathrm{L}\right)$ - and high ( $\left.\geq 1.64 \times 10^{\circ} / \mathrm{L}\right)$-ALC groups.

Note: The RFS rate was significantly higher in the high-ALC group than in the low-ALC group $(P<0.00 \mathrm{I})$.

Abbreviations: RFS, recurrence-free survival; ALC, absolute lymphocyte count.

that maintaining ALC in RHCC patients might improve outcomes.

\section{Acknowledgments}

This study was supported by the National Scientific Foundation Committee of China (81127006 and 81071210) and the International Cooperation Plan of National Science and Technology Department of China (2012DFG32070).

\section{Disclosure}

The authors report no conflicts of interest in this work.

\section{References}

1. Knudsen ES, Gopal P, Singal AG. The changing landscape of hepatocellular carcinoma: etiology, genetics, and therapy. Am J Pathol. 2014;184:574-583.

2. Siegel R, Naishadham D, Jemal A. Cancer statistics, 2013. CA Cancer J Clin. 2013;63:11-30.

3. Aravalli RN, Steer CJ, Cressman EN. Molecular mechanisms of hepatocellular carcinoma. Hepatology. 2008;48:2047-2063.

4. Faber W, Stockmann M, Schirmer C, et al. Significant impact of patient age on outcome after liver resection for HCC in cirrhosis. Eur J Surg Oncol. 2014;40:208-213.

5. Lencioni R. Loco-regional treatment of hepatocellular carcinoma. Hepatology. 2010;52:762-773.

6. Lupo L, Panzera P, Giannelli G, Memeo M, Gentile A, Memeo V. Single hepatocellular carcinoma ranging from 3 to $5 \mathrm{~cm}$ : radiofrequency ablation or resection? HPB (Oxford). 2007;9:429-434.

7. Liang P, Yu J, Yu XL, et al. Percutaneous cooled-tip microwave ablation under ultrasound guidance for primary liver cancer: a multicentre analysis of 1363 treatment-naive lesions in 1007 patients in China. Gut. 2012;61:1100-1101.
8. O'Keefe SJ, El-Zayadi AR, Carraher TE, Davis M, Williams R. Malnutrition and immuno-incompetence in patients with liver disease. Lancet. 1980;2:615-617.

9. Nagai S, Abouljoud MS, Kazimi M, Brown KA, Moonka D, Yoshida A. Peritransplant lymphopenia is a novel prognostic factor in recurrence of hepatocellular carcinoma after liver transplantation. Transplantation. 2014;97:694-701.

10. Milne K, Alexander C, Webb JR, et al. Absolute lymphocyte count is associated with survival in ovarian cancer independent of tumorinfiltrating lymphocytes. J Transl Med. 2012;10:33.

11. Riesco A. Five-year cancer cure: relation to total amount of peripheral lymphocytes and neutrophils. Cancer. 1970;25:135-140.

12. Ray-Coquard I, Cropet C, Van Glabbeke M, et al. Lymphopenia as a prognostic factor for overall survival in advanced carcinomas, sarcomas, and lymphomas. Cancer Res. 2009;69:5383-5391.

13. Pardee AD, Butterfield LH. Immunotherapy of hepatocellular carcinoma: unique challenges and clinical opportunities. Oncoimmunology. 2012;1:48-55.

14. Zhou P, Liang P, Yu X, Wang Y, Dong B. Percutaneous microwave ablation of liver cancer adjacent to the gastrointestinal tract. $J$ Gastrointest Surg. 2009;13:318-324.

15. Liang P, Wang Y, Yu X, Dong B. Malignant liver tumors: treatment with percutaneous microwave ablation - complications among cohort of 1136 patients. Radiology. 2009;251:933-940.

16. Heagerty PJ, Lumley T, Pepe MS. Time-dependent ROC curves for censored survival data and a diagnostic marker. Biometrics. 2000;56:337-344.

17. Ströhlein MA, Lefering R, Bulian DR, Heiss MM. Relative lymphocyte count is a prognostic parameter in cancer patients with catumaxomab immunotherapy. Med Hypotheses. 2014;82:295-299.

18. Watanabe R, Tomita N, Itabashi M, et al. Peripheral blood absolute lymphocyte/monocyte ratio as a useful prognostic factor in diffuse large B-cell lymphoma in the rituximab era. Eur J Haematol. 2014;92:204-210.

19. Sundar R, Soong R, Cho BC, Brahmer JR, Soo RA. Immunotherapy in the treatment of non-small cell lung cancer. Lung Cancer. 2014;85: 101-109. 
20. Xiang B, Snook AE, Magee MS, Waldman SA. Colorectal cancer immunotherapy. Discov Med. 2013;15:301-308.

21. Matsueda S, Graham DY. Immunotherapy in gastric cancer. World $J$ Gastroenterol. 2014;20:1657-1666.

22. Galon J, Angell HK, Bedognetti D, Marincola FM. The continuum of cancer immunosurveillance: prognostic, predictive, and mechanistic signatures. Immunity. 2013;39:11-26.
23. Chen DS, Mellman I. Oncology meets immunology: the cancerimmunity cycle. Immunity. 2013;39:1-10.

\section{Publish your work in this journal}

OncoTargets and Therapy is an international, peer-reviewed, open access journal focusing on the pathological basis of all cancers, potential targets for therapy and treatment protocols employed to improve the management of cancer patients. The journal also focuses on the impact of management programs and new therapeutic agents and protocols on

\section{Dovepress}

patient perspectives such as quality of life, adherence and satisfaction. The manuscript management system is completely online and includes a very quick and fair peer-review system, which is all easy to use. Visit http://www.dovepress.com/testimonials.php to read real quotes from published authors.

Submit your manuscript here: http://www.dovepress.com/oncotargets-and-therapy-journal 